cerebration and sudden cardiorespiratory arrest may also result from increased intracranial pressure, although direct involvement of the brain stem reticular substance may play a part.? The extent of the spinal cord involvement is remarkable. It is a well-known result of HCP toxicity in rats, ${ }^{8}$ in which paralysis of the hindquarters often occurs, but has been only rarely described in man. ${ }^{4}$ No obvious cerebral sequelae were noted in the 14 surviving children. Follow-up was not long enough, however, to exclude the possibility of more subtle damage, which may be manifested by learning difficulty or behaviour disorder. The asymmetrical ventricular dilatation noted in one patient one month after appearance of the first symptoms suggests that damage may occur in some patients and might eventually become symptomatic.

Our neuropathological results accord with experimental data ${ }^{9}$ and with the findings of Martinez et $a l^{16}$ and Mullick ${ }^{3}$ in infants. The main pathological effect of HCP is intramyelinic oedema without demyelination. The oedema is located within myelin sheaths between lamellae and is unaccompanied by disturbances of the blood-brain barrier. ${ }^{9}$ Brain-stem lesions in our patients were consistent with the findings of Shuman et $a l^{6}$ and Powell et al ${ }^{\circ}$ in premature babies bathed in a $3^{\prime \prime}$, HCP solution. In our cases, however, myelinic oedema was much more widespread, as shown by the large increase in brain weight and the intense lacework oedema in the spinal cord.

The neuropathological findings satisfactorily account for the clinical picture, especially the intracranial hypertension. The lack of demyelination and preservation of neurones probably explain the apparent reversibility of the lesions. Extensive oedema occurring within a rigid structure such as the spinal canal, however, can result in infarction of nervous tissue, as observed in case 6 , thus giving rise to permanent damage. In addition, axonal degeneration has been noted after chronic intoxication in rats ${ }^{17}$ and could be another source of permanent damage in man.

We are indebted to Dr J D Lockhardt (Bureau of Drugs, Food, and Drug Administration, Rockville, Maryland) for blood and CSF determinations of HCP, and to Dr M L Arsenio-Nunes (Hôpital Saint Vincent de Paul, Laboratoire de Neuropathologie, U 154, INSERM, Paris) for the ultramicroscopical examination.

\section{References}

1 Larson, D E, Hospitals, 1968, 42, 63.

2 Lockhart, J D, Pediatrics, 1972, 50, 229.

${ }^{3}$ Mullick, F G, Pediatrics, 1973, 51, 395.

${ }^{4}$ Chilcote, R, et al, Pediatrics, 1977, 59, 457.

5 Powell, H, et al, fournal of Pediatrics, 1973, 82, 976.

'Shuman, R M, Leech, R W, and Alvord, E C, Pediatrics, 1974, 54, 689.

Shuman, R M, Leech, R W, and Alvord, E C, Archives of Neurology, 1975 , 32, 320.

${ }^{\star}$ Kimbrough, R D, and Gaines, T B, Archives of Environmental Health, $1971,23,114$

9 Lampert, P, O'Brien, J, and Garrett, R, Acta Neuropathologica, 1973, 23, 326

10 British Medical fournal, 1977, 1, 337.

${ }^{11}$ Herter, W B, Kaiser Foundation Medical Bulletin, 1959, 7, 228.

${ }^{12}$ Curley, A, et al, Lancet, 1971, 2, 296

13 Kopelman, A E, fournal of Pediatrics, 1973, 82, 972.

${ }^{14}$ Abbott, L M, et al, Australian Paediatric fournal, 1972, 8, 246.

${ }^{15}$ Alder, V G, et al, Lancet, 1972, 2, 384.

${ }^{16}$ Martinez, A J, Boehm, R, and Hadfield, M G, Acta Neuropathologica, 1974, 28, 93.

${ }^{17}$ Rose, A L, Wisniewski, H M, and Cammer, W, fournal of the Neurological Sciences, 1975, 24, 425 .

\title{
Is your dosage really necessary? Antibiotic dosage in urinary infection
}

\author{
DAVID GREENWOOD, FRANCIS O'GRADY
}

British Medical fournal, 1977, 2, 665-667

\section{Summary}

Dense cultures of bacteria were exposed to changing concentrations of beta-lactam antibiotics in an in-vitro model of the urinary bladder.

The results suggest that uncomplicated urinary infection may respond to lower doses than are usually given without compromising the success of subsequent treatment in those cases in which such minimal treatment fails.

\section{Introduction}

There are strong indications that uncomplicated urinary tract infection will respond satisfactorily to less intensive treatment than is usual. ${ }^{12}$ Nevertheless, the optimal dosage for the treat-

\footnotetext{
Department of Microbiology, University of Nottingham, City Hospital, Nottingham NG5 1 PH

DAVID GREENWOOD, BSC, PHD, senior lecturer in microbiology FRANCIS O'GRADY, FRCP, FRCPATH, professor of microbiology
}

ment of bacterial infections of the urine remains to be defined. The laboratory has been of little help in this respect because the conditions in which bacteria and drug interact in the urine are so far removed from those of conventional in-vitro tests that it is difficult to predict the response to treatment.

As an approach to this problem, and to attempt to discriminate between the many agents now available, we have developed an in-vitro model in which bacteria grow in the conditions of dilution and periodic discharge that obtain in the urinary bladder and in which dense bacterial cultures (as are common in infected urine) can be exposed to the changing drug concentrations that are provided by therapeutic regimens.

We describe here our investigation into the effect of penicillins and cephalosporins on Escherichia coli and Proteus mirabilis, the organisms most often associated with urinary tract infection in domiciliary practice. Because of the bewildering variety of $\beta$-lactam antibiotics now being produced, we limited our study to representatives of the group that have distinct properties in terms of their antibacterial activity against urinary tract pathogens.

\section{Method}

An overnight culture of bacteria in "complete" broth ${ }^{3}$ was diluted with fresh broth at $1 \mathrm{ml} / \mathrm{min}$ (the normal diurnal rate of urine flow into the bladder). Every hour the accumulated broth was pumped 
from the bladder model (simulating micturition), leaving a $20-\mathrm{ml}$ residuum. The "bladder" temperature was kept at $37^{\circ} \mathrm{C}$ and the opacity of the culture was continuously monitored photometrically. ${ }^{4} \mathrm{~A}$ changing concentration of antibiotic was introduced into the system with the broth inflow by use of a suitably programmed gradientforming device (MixoGrad; Gilson Medical Electronics). The antibiotic profile used is shown in fig 1 . During the first four hours the culture was diluted with antibiotic-free broth, then the antibiotic concentration rapidly rose to reach a peak of $500 \mathrm{mg} / \mathrm{l}$ after two hours and subsequently fell exponentially until, after 12 hours' antibiotic exposure, dilution was again continued with antibiotic-free broth. The total "dose" over the 12-hour period of antibiotic exposure was about $125 \mathrm{mg}$. A second identical cycle of exposure to antibiotic was started when persisting bacteria had re-established the bacterial population.

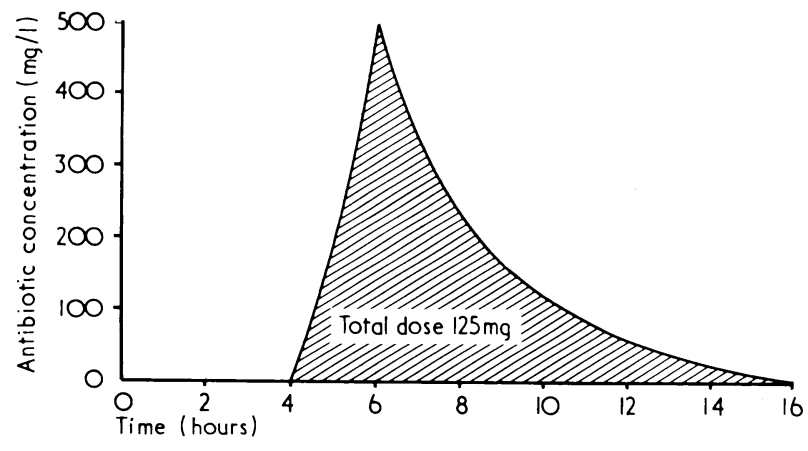

FIG 1-Excretion profile of antibiotic used in the bladder model.

The antibiotics studied were benzylpenicillin, ampicillin, mecillinam, cephalothin, cephalexin, and cefuroxime. In terms of activity against Gram-negative bacilli these represented, respectively, a penicillin of low potency; a penicillin of high potency; an amidinopenicillin of extremely high potency; a cephalosporin of high potency; a cephalosporin of low potency; and a cephalosporin of intermediate potency, which is stable to most enterobacterial $\beta$-lactamases.

\section{Results}

Exposure of the bacterial strains to antibiotic caused a decline in opacity as an inhibitory concentration was achieved. As the antibiotic concentration fell again, persisting bacteria resumed growth and eventually restored the population to its original level. The results were expressed as the time elapsing, after the completion of the 12-hour cycle of drug exposure, for the opacity to reattain a level of half of maximum. These results are presented in fig 2 , which compares the efficacy of the first and second doses of each agent using three urinary



FIG 2-Comparison of ability of sequential doses of $\beta$-lactam antibiotics to suppress bacterial growth in the bladder model. isolates of bacteria (ampicillin-sensitive and ampicillin-resistant $E$ coli and penicillin-sensitive $P$ mirabilis)

A single 125-mg dose of each antibiotic suppressed growth of the ampicillin-sensitive $E$ coli for long periods (21 to 23.5 hours from the start of the dose). Survivors of exposure to ampicillin, benzylpenicillin, cephalothin, and cefuroxime showed a similar susceptibility as the original culture when challenged with a second dose. Survivors of exposure to cephalexin and mecillinam showed a decline in susceptibility, which was dramatic for mecillinam.

A single dose of each antibiotic suppressed growth of the $P$ mirabilis strain for about 24 hours. Sequential exposure of this strain showed that a single cycle of exposure to antibiotic reduced the susceptibility of survivors by about the same amount in all cases.

Penicillins were inactive against the ampicillin-resistant $E$ coli strain. Of the cephalosporins tested, cefuroxime was the most effective and was least affected by sequential exposure.

\section{Discussion}

By using the bladder model, the interaction of bacteria with antibiotics can be studied under conditions that are much closer to real ones than those provided by conventional laboratory tests: a dense bacterial population is exposed to a changing antibiotic concentration and the culture is continually diluted and periodically discharged, as it is in the urinary bladder. In these conditions each of the antibiotics tested suppressed bacterial growth for a similar period, ampicillin and cefuroxime being marginally the most active. Conventional tests of the minimum inhibitory concentration (MIC) of these agents indicated a wide variation in activity. For example, the MIC of mecillinam for the sensitive $E$ coli strain was $0 \cdot 125 \mathrm{mg} / 1$; that of benzylpenicillin for the same organism was $64 \mathrm{mg} / \mathrm{l}$-a 500 -fold difference. Cefuroxime was equally effective against the ampicillinsensitive and ampicillin-resistant strains, no doubt because of its superior resistance to enzymic destruction. ${ }^{5}$

In the model the urine (broth) provided optimal conditions for bacterial growth, and the bacterial population before treatment was held in excess of $10^{*}$ organisms per ml. The model also represented a difficult patient with an impaired ability to empty the bladder ${ }^{6}$ (the residual volume of the model was $20 \mathrm{ml}$, compared with the normal human value of about $1 \mathrm{ml}^{7}$ ). Despite these unpromising conditions, a single 125-mg dose of antibiotic, achieving a transient peak level of only $500 \mathrm{mg} / \mathrm{l}$, suppressed growth of sensitive bacteria for over 20 hours. This suggests that much more modest doses of $\beta$-lactam antibiotics than those commonly used should be effective in controlling many urinary infections.

Such considerations are not restricted to $\beta$-lactam antibiotics. Very low concentrations of sulphonamides effectively suppress the dense bacterial population of the bladder model for as long as the drug concentration is maintained, despite the massive inoculum effect demonstrable in conventional in-vitro tests. ${ }^{8}$ Similar considerations apply to trimethoprim. ${ }^{8}$ Low doses of nalidixic acid also suppress growth for long periods in the bladder model, although the use of this drug is complicated by the emergence of resistant mutants. ${ }^{9}$

Some urinary infections, however, would certainly not respond to such minimal treatment, and an important aim of our investigation was to establish whether bacteria surviving transient exposure to $\beta$-lactam antibiotics would retain their susceptibility when challenged with a fresh dose. With the ampicillin-sensitive $E$ coli, prior exposure to antibiotic appeared to have little or no effect on subsequent treatment, except in the case of cephalexin and mecillinam. These antibiotics are unusual in that they act at separate sites in the bacterial cell, both of which are attacked by most other $\beta$-lactam agents. ${ }^{111}$ Mecillinam suffers the further disadvantage that bacteria surviving its action show phenotypic resistance in that they are able to grow in the presence of the antibiotic though at a reduced rate and in a morphologically abnormal form, ${ }^{12}$ so that they may be very susceptible to natural defences.

Exposure of the $P$ mirabilis strain to each of the antibiotics 
reduced the effectiveness of the second dose. This was probably due to the lower osmotic susceptibility of $P$ mirabilis compared with $E$ coli, ${ }^{13}$ the reduced efficacy of the second dose reflecting the selection of an osmotically stable fraction of the population during the first cycle of drug exposure. The osmolality of the broth used $(325 \mathrm{mmol}(\mathrm{mOsm}) / \mathrm{kg})$ is within the normal range for human urine, which may, however, be much higher. At higher osmolalities $E$ coli is similarly protected and, conversely, at lower osmolalities $P$ mirabilis is more susceptible to antibioticinduced lysis. ${ }^{13}$ High fluid intake has been recommended in the treatment of urinary tract infection on other grounds, ${ }^{\prime}$ but an additional benefit might accrue in treatment with $\beta$-lactam antibiotics because of the consequent reduction in urinary osmolality.

Charlton et $a l^{2}$ successfully treated urinary infection in nonpregnant women in general practice with amoxycillin, $500 \mathrm{mg}$ three times a day for three days, and observed no advantage when treatment was extended for 10 days. Our findings offer microbiological evidence that similar success may be achieved by reducing the dose as well as the length of treatment.

\section{References}

1 Cattell, W R, et al, Urinary Tract Infection, ed F O'Grady and W Brumfitt, p 212. London, Oxford University Press, 1968.

2 Charlton, C A C, et al, British Medical fournal, 1976, 1, 124.

${ }^{3}$ Greenwood, D, and O'Grady, F, fournal of infectious Diseases, 1973, 128, 211

${ }^{4}$ O'Grady, F, et al, British fournal of Experimental Pathology, 1973, 54, 283.

${ }^{5}$ O'Callaghan, C H, et al, Antimicrobial Agents and Chemotherapy, 1976, 9, 511.

6 Shand, D G, et al, Lancet, 1970, 1, 1035

' Shand, D G, et al, British fournal of Urology, 1968, 40, 196.

${ }^{8}$ Greenwood, D, and O'Grady, F, fournal of Clinical Pathology, 1976, 29, 162.

${ }^{9}$ Greenwood, D, and O'Grady, F, Chemotherapy. In press.

10 Greenwood, D, and O'Grady, F, Fournal of Infectious Diseases, 1973, 128, 791.

${ }^{11}$ Spratt, B G, Proceedings of the National Academy of Sciences of the United States of America, 1975, 72, 2999.

12 Greenwood, D, and O'Grady, F, Fournal of Clinical Pathology, 1973, 26, 1.

13 Greenwood, D, and O'Grady, F, British fournal of Experimental Pathology, 1972, 53, 457.

(Accepted 21 fuly 1977)

\title{
Minoxidil for severe hypertension after failure of other hypotensive drugs
}

\author{
B L DEVINE， R FIFE， P M TRUST
}

British Medical fournal, 1977, 2, 667-669

\section{Summary}

Forty-four patients with severe hypertension who were resistant to treatment with more conventional hypotensive drugs or could not tolerate the side effects were treated with minoxidil, a potent peripheral vasodilator. $A$ beta-blocking drug and a diuretic were used routinely to control, respectively, the tachycardia and fluid retention caused by minoxidil. During treatment the outpatient supine blood pressure fell from a mean of $221 / 134 \mathrm{~mm} \mathrm{Hg}$ to $162 / 98 \mathrm{~mm} \mathrm{Hg}$. Eleven patients required additional or alternative hypotensive agents before blood pressure was adequately controlled. Side effects were minor, although the invariable hirsuties caused by minoxidil was unacceptable to three women.

The possibility of cardiotoxic effects, raised by early studies in dogs, has not been excluded, and therefore this drug should be used only in patients with severe hypertension. In such patients minoxidil appears to be most effective.

\section{Introduction}

Minoxidil has recently become available on trial in Britain for

Division of Medicine, Royal Infirmary, Glasgow

B L DEVINE, MB, MRCP, medical registrar

R FIFE, MB, FRCP, consultant physician

MRC Blood Pressure Unit, Western Infirmary, Glasgow

P M TRUST, MB, BS, clinical scientist treating patients with severe hypertension in whom conventional treatment has failed to control blood pressure. ${ }^{1}$ We describe here the use of minoxidil in 44 such patients.

\section{Patients and methods}

All the patients had diastolic blood pressures (fifth Korotkoff phase; the mean of the last three readings in the outpatient department, while sitting or lying) of over $120 \mathrm{~mm} \mathrm{Hg}$ despite treatment with other hypotensive drugs. Previous hypotensive treatment (table I) had been given in full dosage or had produced intolerable side effects.

Ten patients had been in the malignant phase of hypertension, 14 had had a stroke, nine had had congestive cardiac failure, and five complained of angina of effort. Twenty-two patients had renal failure before starting minoxidil; their blood urea concentrations were consistently over $7 \mathrm{mmol} / 1(42 \mathrm{mg} / 100 \mathrm{ml})$ and serum creatinine concentrations over $120 \mu \mathrm{mol} / 1(1.4 \mathrm{mg} / 100 \mathrm{ml})$.

The patients were all admitted to hospital before starting minoxidil and the following values were measured: full blood count including platelets; serum creatinine, urea, and electrolyte concentrations; cardiac enzymes (lactate dehydrogenase, creatine phosphokinase); serum aspartate and alanine aminotransferase and serum bilirubin concentrations; and fasting blood sugar concentrations. An electrocardiogram (ECG) was recorded and a chest radiograph taken. These investigations were repeated at regular intervals. In general hypotensive drugs other than beta-adrenergic receptor blocking agents and diuretics were withdrawn before starting minoxidil. Beta-blockers and diuretics were continued since previous clinical experience ${ }^{2-6}$ had established the need for these drugs to combat, respectively, tachycardia and sodium retention. Except for the diuretic, which was given once in the morning, other drugs were taken twice daily. Minoxidil was started at a dose of $2.5 \mathrm{mg}$ on the first day and increased over four days to $40 \mathrm{mg}$ if required.

The blood pressure, measured with a traditional clinical sphygmomanometer, and pulse rate were recorded while supine, standing, and again while standing after exercise before each dose of minoxidil was given. The dose was not increased if the diastolic blood pressure fell below $90 \mathrm{~mm} \mathrm{Hg}$. 